\title{
Solar Simulated Light
}

National Cancer Institute

\section{Source}

National Cancer Institute. Solar Simulated Light. NCI Thesaurus. Code C123651.

The therapeutic use of a light source that approximates the characteristics of natural sunlight with regard to the radiation spectrum represented as well as the relative contributions of the wavelengths of the spectrum. Artificial sunlight is used to treat seasonal affective disorder and jet lag and may have other beneficial effects on health. 